\title{
Study on Application of Computer Simulation Technology in Physical Education
}

\author{
Bai Zhen ${ }^{1}$ a, JIn Lei ${ }^{1 b}$ * \\ (1 Research Institution of Students' Physical Health in Henan University, Kaifeng 475001 China ) \\ aemail: 13598757005@163.com, bemail:454622113@qq.com \\ *JIn Lei is the corresponding author of this paper.
}

Key words: computer simulation, physical education, physical research

\begin{abstract}
This paper discusses the concept and characteristics of the computer simulation technology, and the general process of the simulation design, puts forward the problems in applying the computer simulation technology in the physical education, and analyzes the development prospect and significance of applying the computer simulation technology in the physical education.

With the development of the computer science and technology, the computer simulation technology has already been widely applied in the analysis, design, experiment, evaluation, and prediction of the complex system. The model established by the computer simulation system includes the internal correlation and objective law between the actual systematic objects. Through the computer simulation experiment, it is feasible to study the mutual relationship of the internal factors in the system. In the physical science research domain, with the mutual integration and boosting of the system simulation technology, related computer graphics, database technology, and the virtual reality technology, as well as the boosting of the strong demands of the different domains centered on the body or its movement, the virtual body and its movement has already become a hot spot in the current research development. Therefore, to apply the computer simulation technology in the physical science domain has great impact on the physical science study. It will become an important supplementary means in the physical education and competition training.
\end{abstract}

\section{Overview of computer simulation technology}

Computer simulation technology and its role in the teaching and study

In fact, the computer simulation technology is to establish simulation model and carry out the simulation experiment. It carries out the research, analysis and experiment through establishing the model of the research system and based on the environmental (actual or simulated) conditions. As a research method and experiment technology, it is directly applied in the system research. The relationship among the three elements of the computer simulation can be described by 3 basic activities (see Fig. 1). With the development of the computer technology, the computation speed and processing capability of the digital computer is being increased constantly. The digital computer simulation has gradually replaced the analog computer simulation and digital-analogue simulation. Therefore, the current computer simulation generally refers to the digital computer simulation[1]. Its main characteristics are as follows: easy to be realized and modified, low cost, high accuracy, rapid operating rate, and reliable experiment result. 


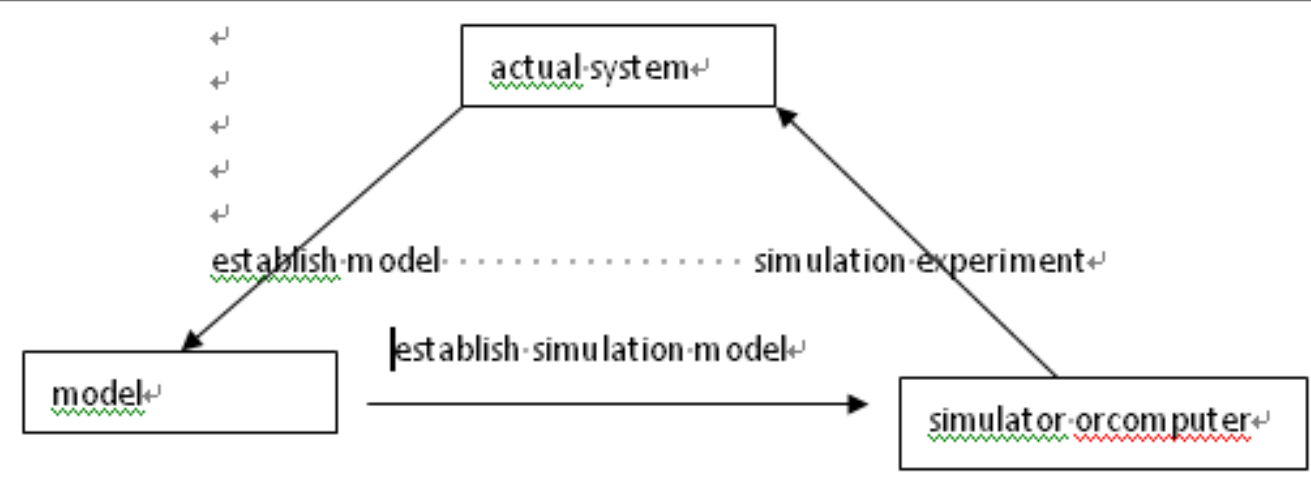

Figure 1 Relations among the Three Key Factors of computer Simulation

Application of computer simulation technology

To apply the computer simulation technology has long reaction period. Besides, it is difficult to observe, test or consume the systems with vast capital, such as natural environment, ecosystem, population structure, physiology, breeding, guided missile, national defense, etc. It can carry out the analysis and post-test forecasting results on the model in short term, such as TCM diagnosis, agriculture breeding expert system, etc.

With regard to the engineering system, to apply the computer simulation technology for the test or pre-research can carry out the demonstration on the systematic research proposal before the entity experiment, to optimize the proposals and systematic parameters; analyze the system response and parameter relationship guidance debugging; analyze the systemic working status and improve the weak links to enhance the performance and operation efficiency of the system.

With regard to the non-engineering system, it is feasible to apply the computer simulation technology on the enterprise management, economic analysis, market forecasting, commodity sales, etc. For instance, to apply this technology can establish the commodity production, corporate management and market forecasting model.

Process of computer simulation

In general, the process of the computer simulation includes three basic parts: "mathematical modeling”, "computer simulation experiment", and "experiment result analysis". That is to say, the computer simulation not only tests the model. It contains the whole process from the mathematical modeling, repeated experiment to the result analysis. The process generally includes the following steps. (1) Problem description: through the problem description, clarify the object, purpose and basic demands of the computer simulation, and determine the scale and constraint condition of the computer simulation system according to the purpose and demands of the research. (2) Establish original mathematical modeling. The mathematical model is a mathematical structure expounded through the mathematical language by referring to the feature or quantity dependence relationship of some object system. The computer simulation is a new technology based on the model. The target degree of the mathematical modeling plays an important role in the reliability of the simulation result. Such step is quite significant. The original mathematical model is also named as the first modeling. (3) Establish analogue-systematic mathematical modeling. According to the computation feature, simulation way, computation method and accuracy requirements of the computer, transform the original systematic mathematical model into the mathematical model whose program design can be realized by the computer. Such model is named as analogue system mathematical model and also secondary modeling. (4) Programming and debugging. Debug the formulation process of the analogue systematic mathematical model. (5) Simulation experiment. Apply the computer simulation system to carry out the specific experiment, and obtain the result. (6) Test result analysis and model verification. After repeating the simulation experiment, analyze and verify the simulation results, select the optimal proposal or find out the optimal value, check the result and actual data, and then verify the accuracy of the simulation system model[2]. 


\section{Application of computer simulation technology in the physical science domain}

Application of computer simulation technology in the physical education domain

With the development of the multi-media technology, it is feasible to enrich the classroom teaching content through applying the simulation multi-media courseware in the theoretical teaching, arouse the students' interest in the learning, enhance their understanding of the theoretical knowledge that is difficult to be understood. On the other hand, during the academic teaching process, most explanation of the content is completed through the teachers' demonstration. However, due to the increasing of the teachers' age or the different skill mastering degrees of the teachers, some of them find difficulty in finishing the correct and standard demonstration in the teaching and training, which has some impact on the students' learning effect. Besides, it is difficult to provide actual evaluation on the students' learning effect. If we can apply the multimedia simulation technology and visualized simulation technology to develop and design some simulation software, and enable the students to obtain cognition and training in the virtual environment, it is feasible to improve the students' study and training effect.

Application method of computer simulation technology in the martial art routine teaching

The martial art routine is unique in the China, and it is the treasure in the Chinese traditional culture, which focuses on the combination of shape, spirit, movement and silence, and the unification of the hands, eyes, body and steps. The current universities have the routine lessons. The related general teaching only focuses on explaining the movement, as well as the teachers' demonstration. However, due to the increasing of the teachers' age and the weakening of their technique and physical quality, they are difficult to complete the demonstration in the teaching and training. In general, the correct movement is realized through the learners' repeated exercise and teachers' constant feedback. In addition, it is important to master the steps of the martial art. However, during the exercise, the students only focus on the proficiency but neglect the steps and position. The problems can be solved through these simulation techniques. We can supplement the teaching through making the simulation software. During the teaching and training, to display the technical movement through the virtual simulation technology not only enables the athletes to master the movement more easily and rapidly,but also improves their technique proficiency. The main ways are as follows[3].

Supplementary demonstration

To introduce the computer simulation technology in the practice lesson not only can enhance the students' interest in learning, but also can better solve the influence of the moment and no supporting point during the movement completion in the demonstration effect, as well as the influence of the limitation of the teachers' profession and the age in demonstrating the movement. For instance, in the martial art teaching, it is feasible to make some simulation multimedia courseware. We can display some complex movement and difficult movement after the teacher shows simple movement. Meanwhile, with the teachers' explanation, the teaching effect can be improved[4].

Process control

In the training site, we place sensing devices under the carpet in the beginning, medium, each turning and ending steps according to the martial art routine. These devices are connected to the computer. Set the time of arriving at each sensing device and interval time. The reaction of each sensing device is displayed on the computer screen at real time. Besides, after the students' training ends up, repeat the demonstration, and promptly make modification if the position is not correct, and the time is mastered improperly. For instance, when practicing Tai Chi routine, the beginners do it fast. We can set several points. Each point is connected to the computer. After the subject touches some point, the computer starts to count. Meanwhile, the red light connected with the computer lights up. The subject stops. After getting to the preset time, the green light lights up. The subject continues the movement. Through a series of the control of the red light and green light, it is feasible to control the rhythm and time of the whole routine (shown in Fig.2). 


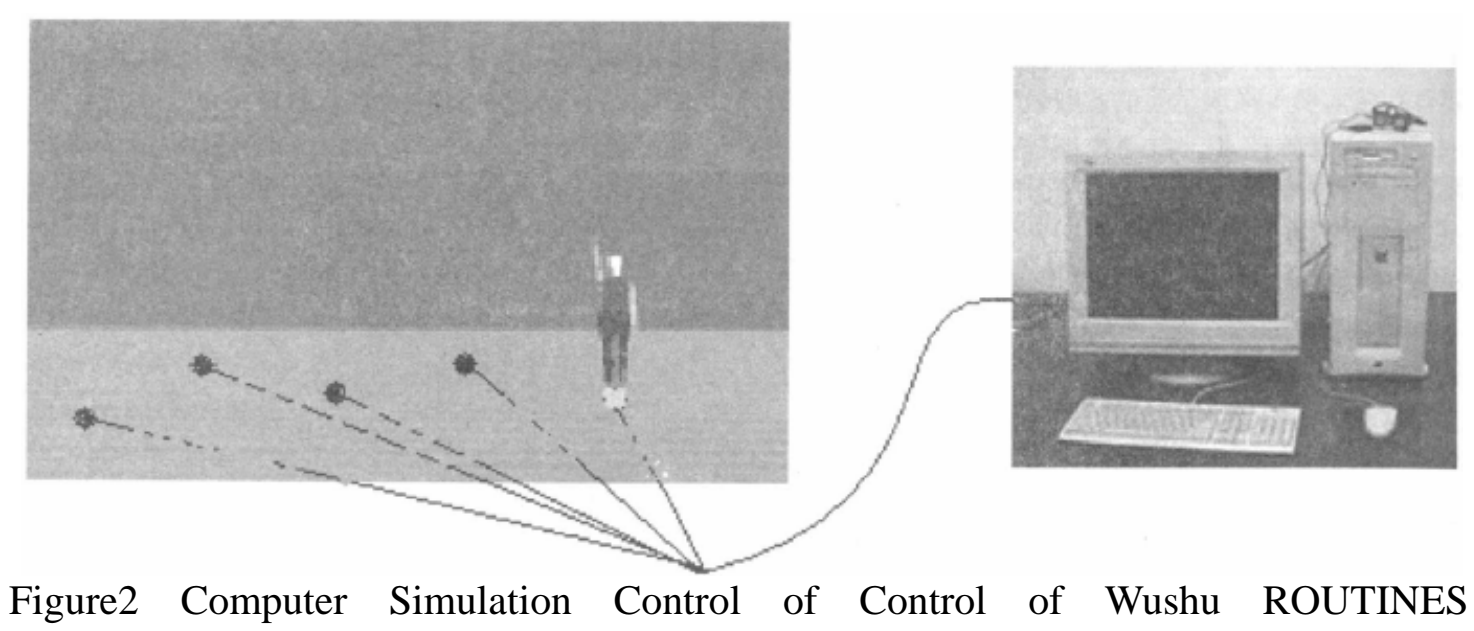

\section{Simulation exercise}

Establish virtual analog figure and virtual environment. The martial art routine is complex and variable. To apply the simulation technique enables the students to virtualize an opponent, and show them through the vivid 3D animation simulation technology. In this way, the learners can observe the tactics feature of the opponent vividly, and then they can better understand their ability, which is favorable for them to improve the learning ability[5].

\section{Problems and prospect}

To apply the computer simulation technology in the physical education and research domain is required to meet some conditions. At present, some problems are worthy of consideration. First of all, due to the complexity of the physical education content, there is no specific computer simulation software aiming at the physical education domain. Secondly, the profession and complexity of the computer simulation technology causes some difficulties to the sports teachers and physical researchers when applying this technology. The solution to these problems requires the researchers to combine physical training and computer simulation technology[6]. For instance, the computer professionals can master some knowledge related to the sports, or the physical researchers and computer professionals can cooperate with each other. Therefore, how to better apply the computer simulation technology in the physical education and science is worthy of thinking and studying. The application research of this technology plays an active and boosting role in the physical education and guiding the sports training.

\section{Conclusion}

The simulation technology is not only aimed at some motion or pure simulation. Instead, through the simulation experiment, it studies the relationship among the internal factors of the system, analyzes the feasibility of the system, forecasts the operation result of the system, and lays solid foundation for the reality system as the pre-research means. The development of the computer simulation technology brings new opportunities for the sports training and physical education research, which has great impact on the development of the physical science research and training mode. We believe, as a new teaching platform, the simulation and simulation technology with its strong teaching, scientific advantage and potential will be gradually paid close attention to by the physical teachers and will become popular. Finally, it will be widely applied in the physical education domain and play an important role in this field. 


\section{References}

[1] Huang Hansheng, Zhu Changyi. Theoretical Basis of Human Motion Computer Simulation [J]. Journal of Guangzhou Sports University, 2007, 27 (5): 1-4.

[2] Wu Xuguang, Wang Xinmin. Computer Simulation Technology \& Application [M]. Xi'an: Northwestern Polytechnical University Press, 1998.

[3] Yan Guodong, Sun Jinhai. Application of Computer Simulation Technology in Physical Education Domain [J]. Sports Science \& Technology, 2008, 29 (5): 68-70.

[ 4] SIMI Realit y M otion Sys tems GmbH. SIMI M ot ion UserManual[ CP/ DK] . 2002.

[5] Zhou Xinglong, Zhao Fang, Sequence motion image acquisition and the development of video analysis system [C], the Tenth National Sports Biomechanics Academic Exchange Conference, 2002.

[6] LiuXiao-Wei. Image processing technology [ M ] . BeiJing: QingHua University Press, 2004. 